\title{
Quantitative data as a clue to auxiliary contraction processes
}

\author{
Laurel MacKenzie, University of Pennsylvania
}

\section{Introduction}

The variable contraction of English auxiliary verbs (e.g. John is $\sim$ John's coming) has been well studied (Zwicky, 1970; Kaisse, 1983; Anderson, 2008, a.o.). However, outside of the sociolinguistic domain, analyses of this phenomenon have been based on judgments rather than on quantitative data. In this paper, I show that considering the quantitative distributions of contracted forms results in a more nuanced picture of contraction, one that has implications for how the process must be situated in the grammar. Specifically, I present data from a corpus study of contraction that support a two-stage analysis of the phenomenon: an allomorphic alternation governs the insertion of contracted and uncontracted forms; then, subsequent phonetic and phonological processes operate on the inserted allomorph.

\section{Setting up the issues}

Early work on contraction (e.g. Zwicky, 1970) modeled full forms (e.g. [hæv, Iz, wil], as opposed to $[\mathrm{v}, \mathrm{z}, \mathrm{l}])$ as the sole underlying form of an auxiliary. Contraction was then attributed to the application of variable phonological processes (deletion of initial consonant, deletion of vowel) on these full forms, to output contracted variants. However, later work, beginning with Kaisse (1983), moved away from this strictly phonological account of contraction to one in which full and contracted forms are distinct allomorphs underlyingly, with a variable morphosyntactic process of host-auxiliary adjunction conditioning the insertion of those contracted forms. This analysis will be adopted here.

A number of questions concerning this allomorphic analysis still remain unanswered, though. For instance, what factors condition the application of the proposed adjunction process? And are those conditioning factors the same for each full/contracted pair, or are they auxiliary-specific? In other words, is there a single pan-auxiliary rule of contraction that applies regardless of auxiliary identity, as Rickford et al. (1991) proposed for is and are? Or is "contraction" instead a set of fragmented, auxiliary-specific processes, each with its own variable conditions on application?

Quantitative data will provide evidence of the factors conditioning this variation, and is thus crucial for determining how the process of auxiliary contraction must be represented in the grammar. To that end, I carried out the corpus study described below.

\section{Methodology of the corpus study}

I examined 1075 tokens of the auxiliaries has, have, is, and will collected from the Switchboard corpus, a corpus of 2400 five-minute telephone conversations between strangers (Godfrey et al., 1992). Only tokens in which the auxiliary followed a non-pronoun subject were examined for this study.

In coding the dependent variable of auxiliary realization, a tripartite coding scheme was adopted in order to accurately capture all surface forms that occurred in the data. Tokens were coded as full (1a), intermediate (1b), or contracted (1c). (Henceforth, I will use the terms "full," "intermediate," and "contracted" to refer to forms of the (1a), (1b), and (1c) types, respectively.) 
(1) a. Full: John $\underline{[\mathrm{hæz}] /[\mathrm{həz}]}$ been there all day. (initial consonant, audible vowel of any quality)

b. Intermediate: John [əz] been there all day. (no initial consonant but audible vowel)

c. Contracted: John $\underline{\underline{z}]}$ been there all day. (no initial consonant, no vowel: a single consonant that forms a syllable with its host)

So-called "intermediate" forms have received little attention in the literature on contraction. This is partly due to the fact that those researchers carrying out empirical studies of contraction (e.g. Labov, 1969) have focused almost primarily on the contraction of is, for which intermediate forms are irrelevant. But even where auxiliaries other than is have been examined with quantitative data (e.g. McElhinny, 1993), researchers have brushed intermediate forms to the side or omitted them entirely.

However, intermediate forms turn out to be well-represented in the corpus data, and a comprehensive analysis of the contraction process must take them into account. Before moving on to a discussion of how contraction must be represented in the grammar, then, I propose an analysis of intermediate forms.

\section{Analysis of intermediate forms}

I have identified two plausible sources of intermediate forms.

- Full forms that have lost their initial consonant. A process of initial-consonant deletion, combined with a process of vowel reduction (common among function words), could output surface intermediate forms. In fact, there is an independently-attested (see Kaisse, 1985) process of /h/-Deletion in English, which deletes / h/ when it begins unstressed syllables. This affects pronouns and function words in conversational speech (e.g. he, him), and could thus account for any intermediate forms of has, have found in the data.

However, I follow Kaisse in assuming that there is no comparable fast-speech deletion of $/ \mathrm{w} /$ that would affect will. Intermediate forms of will thus need another explanation.

- Contracted forms with an epenthetic schwa. The auxiliary will is found to surface in its contracted form at a high rate (91\%) after vowel-final pronouns (e.g. he, I, etc.) After the pronoun it, on the other hand, contracted forms are not found (unsurprisingly, given English phonotactics), but intermediate forms are found instead, at a comparably high rate $(79 \%)$. This near-complementary distribution is easily explained if we allow that intermediate forms may derive from underlyingly contracted forms that undergo a repair process of [ə]-Insertion when they cannot syllabify with their host.

This failure to syllabify need not be strictly for phonotactic reasons. Contracted forms of non- $\{$ is, has $\}$ auxiliaries also fail to syllabify with their host when the host is a nonpronoun noun phrase, regardless of phonotactic acceptability (e.g. *Sue'll [sul] 'Sue will', *three've [ $\theta$ riv] 'three have'). The alternative to full forms for these auxiliaries in such cases is, again, intermediate forms. So once again, I analyze these as cases where a contracted allomorph is inserted, but fails to syllabify, necessitating a schwa-insertion repair process.

This analysis of intermediate forms maintains an underlying bipartite distinction (between full and contracted), despite there being a tripartite distinction on the surface. Table 1 summarizes the source of each auxiliary's surface forms, after non-pronoun subjects only. 


\begin{tabular}{l|llll} 
& is & has & will & have \\
\hline \hline $\begin{array}{l}\text { Underlying contracted } \\
\text { forms surface as: }\end{array}$ & contracted & contracted & $\begin{array}{c}\text { intermediate } \\
\text { (from [ə]-Ins.) }\end{array}$ & $\begin{array}{c}\text { intermediate } \\
\text { (from [ə]-Ins.) }\end{array}$ \\
\hline $\begin{array}{l}\text { Underlying full forms } \\
\text { surface as: }\end{array}$ & full & $\begin{array}{c}\text { full \& interm. } \\
\text { (from } / \mathrm{h} / \text {-Del.) }\end{array}$ & full & full \& interm. \\
(from $/ \mathrm{h} /$-Del.)
\end{tabular}

Table 1: Source of surface forms after non-pronoun subjects for all auxiliaries under study.

For the auxiliaries is, has, and will, each surface form maps unambiguously to an underlying form. Surface contracted forms of is and has are underlyingly contracted, surface intermediate forms of has are underlyingly full (having undergone /h/-Deletion), and surface intermediate forms of will are underlyingly contracted (having undergone [ə]-Insertion). (Surface full forms of all three auxiliaries are underlyingly full.) But under the analysis proposed here, surface forms of have do not map neatly onto a single underlying source. This is because intermediate forms of have are a hybrid class: some are underlyingly contracted forms that have had [ə] epenthesized for syllabification, while others are underlyingly full forms that have lost their initial $/ \mathrm{h} /$. The next section presents further evidence from the corpus study in favor of this analysis.

\section{Implications}

If contraction is best represented as a single, pan-auxiliary alternation between two allomorphs, any factors conditioning that alternation should govern the appearance of an auxiliary's surface realizations of full and contracted forms, as laid out in the table above. In order to test this analysis, I examined the conditioning factor of the weight of the noun phrase to which the auxiliary attaches. The hypothesis under examination is that contraction - whatever its surface manifestation - will be disfavored with larger or longer subjects (see Sells, 1983 for a related proposal).

All tokens of auxiliaries after non-pronoun subjects were coded for the number of syllables in the subject. If contraction is disfavored with larger or longer subjects, then an auxiliary's surface manifestation of its underlying contracted form should be less commonly used with bigger subjects.

The plots in Figure 1 show full forms opposed to (the hypothesized surface manifestation of) contracted forms, for subjects of increasing numbers of syllables. The auxiliaries is, has, and will show clearly that as the number of syllables in a subject increases, the number of contracted forms observed with a subject of that length decreases, to the point that above a 10 -syllable subject, contraction is virtually nonexistent. The one graph that differs from the others is that for have, which shows no effect of subject length when full forms are opposed to intermediate forms. This is unsurprising given the analysis proposed here, under which intermediate forms of have are a hybrid of underlyingly full and contracted forms. There is no way to separate the two underlying sources of have's intermediate forms on the surface.

In other words, those three auxiliaries for which contracted forms can be unambiguously distinguished from full all show the same effect of subject weight. These findings not only support the analysis of intermediate forms presented here, but also provide evidence for a model of contraction as a single, pan-auxiliary process, with conditions on its application that affect all auxiliaries to which it applies. 

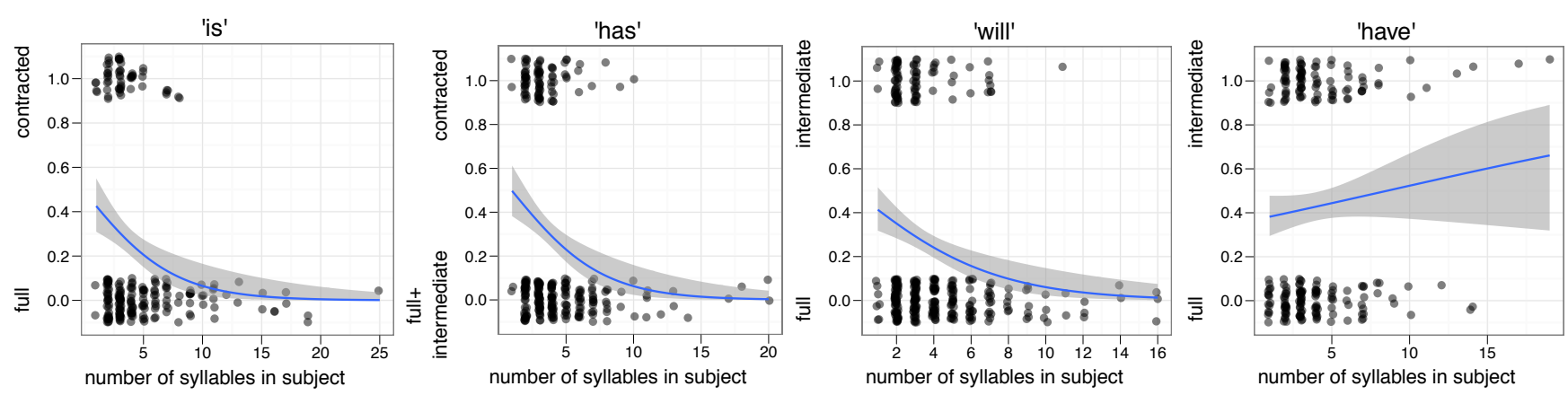

Figure 1: Negative correlation between length of subject and rate of contraction.

\section{Conclusions}

This paper has shed new light on the familiar variable of English auxiliary contraction, as follows. I have documented and analyzed intermediate forms, which are highly prevalent, but have been overlooked in the literature. I have found evidence for an effect of subject weight on the alternation between full and contracted forms. And I have argued that my findings are best analyzed by identifying two stages of processes that affect auxiliary realization: an allomorphic alternation between full and contracted forms, followed by subsequent phonological or phonetic processes that obscure the underlying identity of those forms. Future work will investigate the precise nature of the subject weight effect (is it due strictly to syllable count, or instead to words or syntactic nodes? Why should contraction show such an effect?) and explore how best to represent this effect in the grammar.

\section{References}

Anderson, Stephen R. 2008. English reduced auxiliaries really are simple clitics. Lingue e Linguaggio 7:169-186.

Godfrey, John J., Edward C. Holliman, and Jane McDaniel. 1992. SWITCHBOARD: Telephone speech corpus for research and development. In Proceedings of the IEEE International Conference on Acoustics, Speech, and Signal Processing, Volume 1, 517-520.

Kaisse, Ellen M. 1983. The syntax of auxiliary reduction in English. Language 59:93-122.

Kaisse, Ellen M. 1985. Connected Speech: The Interaction of Syntax and Phonology. New York: Academic Press.

Labov, William. 1969. Contraction, deletion, and inherent variability of the English copula. Language 45:715-762.

McElhinny, Bonnie S. 1993. Copula and auxiliary contraction in the speech of White Americans. American Speech 68:371-399.

Rickford, John R., Arnetha Ball, Renée Blake, Raina Jackson, and Nomi Martin. 1991. Rappin on the copula coffin: Theoretical and methodological issues in the analysis of copula variation in African-American Vernacular English. Language Variation and Change 3:103-132.

Sells, Peter. 1983. Juncture and the phonology of auxiliary reduction in English. In University of Massachusetts Occasional Papers in Linguistics 8, ed. Toni Borowsky and Daniel Finer, 76105.

Zwicky, Arnold M. 1970. Auxiliary reduction in English. Linguistic Inquiry 1:323-336. 